\title{
Assessing the feasibility of dietary soybean meal replacement for fishmeal to the swimming crab, Portunus pelagicus, juveniles
}

\begin{abstract}
The feasibility of dietary soybean meal (SBM) replacement for fishmeal was evaluated to the swimming crab Portunus pelagicus juveniles over six consecutive molts by measuring their growth, development, hemolymph cholesterol, triglycerides, phosphate, whole-body crude protein and cholesterol as well as hepatopancreatic trypsin activity, histopathology and glycogen reserves. A total of six isonitrogenous, isolipidic and isoenergetic diets were formulated with SBM replacing fishmeal at 0 (control), 20, 40, 60, 80 or $100 \%$ of total dietary protein. Each treatment consisted of 30 replicate crabs starting from the first juvenile stage and after the crabs molted to the seventh stage, three-day post molt crabs were sampled. Results showed that crabs fed the 20\% SBM diet had the best growth, which was significantly higher than the control diet $(0 \% \mathrm{SBM})$. Growth became significantly lower in the $60 \%$ dietary SBM treatment and above, while hemolymph cholesterol, triglycerides and phosphate significantly decreased with increasing dietary SBM. The whole-body moisture, crude protein and cholesterol were unaffected by dietary SBM. Meanwhile, hepatopancreatic trypsin activity significantly decreased in the 20 to $60 \%$ SBM treatments, with a further significant decrease in the 80 and $100 \%$ SBM treatments. Hepatopancreatic damage, significantly fewer epithelial cells and glycogen reserves occurred at 60\% SBM and above. Results indicate that dietary SBM can replace up to $40 \%$ of fishmeal in the diets of P. pelagicus juveniles without reducing their growth or hepatopancreatic condition.
\end{abstract}

Keyword: Trypsin; Hepatopancreatic histopathology; Glycogen; Fish meal replacement; Portunus pelagicus 